\title{
Wideband Extrapolation of Spatial Responses of Resonant Structures Using Early-Time and Low-Frequency Data
}

\author{
J. Michael Frye ${ }^{1}$ and Anthony Q. Martin ${ }^{2}$ \\ ${ }^{1}$ The Johns Hopkins University Applied Physics Laboratory, 11100 Johns Hopkins Road, Laurel, MD 20723, USA \\ ${ }^{2}$ The Holcombe Department of Electrical and Computer Engineering, Clemson University, Clemson, SC 29634-0914, USA \\ Correspondence should be addressed to J. Michael Frye; james.frye@jhuapl.edu
}

Received 30 April 2013; Revised 27 August 2013; Accepted 29 August 2013

Academic Editor: Xavier Ferrieres

Copyright (c) 2013 J. M. Frye and A. Q. Martin. This is an open access article distributed under the Creative Commons Attribution License, which permits unrestricted use, distribution, and reproduction in any medium, provided the original work is properly cited.

\begin{abstract}
An efficient procedure is presented to extrapolate a wideband electromagnetic response defined over an arbitrary spatial region using early-time and low-frequency data. The previous procedures presented in the literature are efficient for single-point extrapolation and can readily be applied to spatial regions but are terribly inefficient when a response is desired at many spatial locations. In this work, an optimized algorithm is presented to quickly extrapolate over a large number of spatial locations. The time and frequency behavior of the response is fitted by polynomials and pole terms, and the spatial variation is represented with spatially dependent polynomial coefficients and pole residues. A single set of poles, common to all spatial locations of interest, is shown to sufficiently describe the resonant behavior of response over the entire spatial region. A multisignal formulation of the matrix pencil method is applied to determine poles from early time data. Numerical examples are presented to demonstrate the procedure. Additionally, an automated approach to distinguish physical poles, which correspond to structural resonances, from nonphysical fitting poles is presented. The spatially dependent residues of physical pole terms, referred to here as modal residues, are shown to provide important insight into the resonant behavior of a structure.
\end{abstract}

\section{Introduction}

In [1-4], electromagnetic responses, such as the driving-point current of an antenna, are simultaneously extrapolated in time and frequency by fitting discrete values of the response evaluated at early time and low-frequency points. Early-time and low-frequency data are mutually complementary and together can provide all the information needed to characterize the complete response [1-4]. Determining a wideband response of a resonant structure with computational electromagnetic (CEM) methods can be burdensome; however, extrapolation can significantly reduce the computational load since the complete response is not determined exclusively in either domain $[1,2]$.

An efficient and reliable procedure is presented here to extrapolate a response defined over an arbitrary spatial region, such as a contour, surface, or volume. As in $[1,2]$, the time and frequency behavior of the response is fitted by the sums of polynomials and pole terms. It is shown here that the spatial variation of a response can be accurately represented with sets of spatially-dependent coefficients for the polynomials and spatially-dependent residues for the pole terms. Additionally, it is demonstrated that a single set of poles, shared by each spatial location, is sufficient to describe the resonant behavior of response over the entire spatial region.

As with the procedures of [1-4], the reliable application of the approach in this work requires the selection of several polynomial and pole-estimation parameters. An automated genetic algorithm- (GA-) based procedure is presented in [2] to select the parameters needed to extrapolate a response. To extrapolate a response in a spatial region, one can apply the automated, optimization-based procedure of [2] at each discrete location; however, this approach is highly inefficient because a separate GA optimization run is required for each location. Depending on the size and desired spatial resolution of the region of interest, there could be hundreds or thousands of discrete locations. Conversely, the procedure in this work only requires a single optimization to extrapolate a spatial response. 
All necessary parameters are selected using a GA by simultaneously fitting the response, in early time and low frequency, at a small subset of the total number of positions in the spatial region. Using the parameters selected, a single set of poles is determined with a multisignal formulation of the matrix pencil method (MPM) [5]. Then coefficients and residues are easily calculated for each spatial location by fitting the early-time and low-frequency data at each location. The response is accurately extrapolated over the entire spatial region despite selecting parameters based on data at only a few locations. In addition to being more computationally efficient the procedure in this work is preferred because it yields a representation of the spatial response as a single set of poles and polynomials whereas applying procedure of [2] at each spatial location does not.

A new procedure is also presented to reliably distinguish between physical poles, which correspond to structural resonances, and fitting poles, which are not related to resonances but can improve fitting. Estimating poles from numerical data with techniques such as MPM typically yield fitting poles along with physical poles. Distinguishing between physical and fitting poles is generally difficult (e.g., see [6$8]$ ), but the procedure in this work automates this process. Identifying physical poles is of great interest because they provide important information about the resonant behavior of a structure or system. Additionally, it is shown that the spatial residues of physical pole terms, referred to here as modal residues, correspond to natural modal behavior.

\section{Extrapolation of a Spatial Response}

Let $x(\mathbf{r}, t)$ and $X(\mathbf{r}, f)$ denote the time- and frequencydomain representations, respectively, of an EM response due to a wideband excitation source at a spatial position $\mathbf{r}$. The response corresponds to an EM quantity defined in a spatial region $\mathscr{R}$ which could be, for instance, a contour, surface, or volume.

The early-time and low-frequency ranges of $x(\mathbf{r}, t)$ and $X(\mathbf{r}, f)$ contain high-frequency and late-time information, respectively, and can be used to determine an accurate representation of the complete response. Pole terms can efficiently represent resonant behavior, whereas compact-support polynomials are well-suited to characterize nonresonant behavior [2].

The responses $x(\mathbf{r}, t)$ and $X(\mathbf{r}, f)$ are represented by $\widehat{x}(\mathbf{r}, t)$ and $\widehat{X}(\mathbf{r}, f)$, which are defined as

$$
\begin{gathered}
x(\mathbf{r}, t) \approx \widehat{x}(\mathbf{r}, t)=\sum_{n=0}^{N-1} a_{n}(\mathbf{r}) \phi_{n}\left(\frac{t}{\ell_{1}}\right)+\sum_{m=0}^{M-1} R_{m}(\mathbf{r}) g_{m}(t), \\
X(\mathbf{r}, f) \approx \widehat{X}(\mathbf{r}, f)=\sum_{n=0}^{N-1} a_{n}(\mathbf{r}) \Phi_{n}\left(\frac{f}{\ell_{2}}\right)+\sum_{m=0}^{M-1} R_{m}(\mathbf{r}) G_{m}(f),
\end{gathered}
$$

for $\mathbf{r} \in \mathscr{R}$. In (1), $\phi_{n}\left(t / \ell_{1}\right)$ and $\Phi_{n}\left(f / \ell_{2}\right)$, denote $n$ th-order polynomials which are scaled by factors $\ell_{1}$ and $\ell_{2}\left(\ell_{2}=\right.$ $\left.1 /\left(2 \pi \ell_{1}\right)\right)$ [9], related by the Fourier transform, and each weighted by a spatially-dependent, real-valued coefficient $a_{n}(\mathbf{r})$. The $m$ th pole term is defined as $g_{m}(t)=\exp \left(s_{m} t\right)$ in

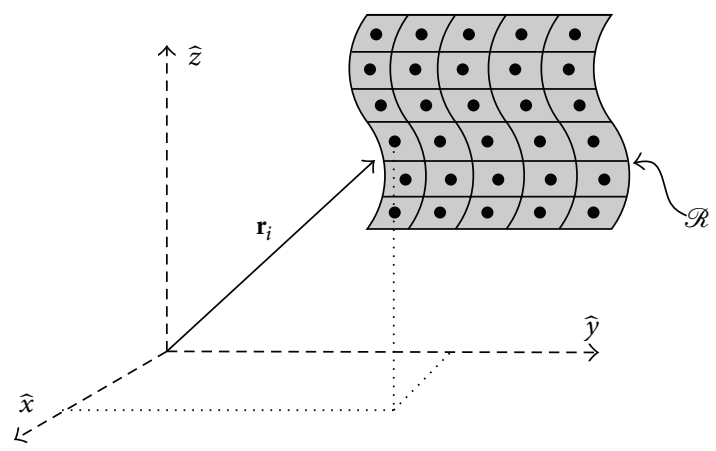

FIGURE 1: Illustration of region of interest $\mathscr{R}$ and discrete spatial location $\mathbf{r}_{i}$.

the time domain and as $G_{m}(f)=1 /\left(j 2 \pi f-s_{m}\right)$ in the frequency domain. Each pole term is weighted by a spatiallydependent, complex residue $R_{m}(\mathbf{r})$. The $m$ th complex pole is $s_{m}=\alpha_{m}+j 2 \pi f_{m}$, where $\alpha_{m}$ and $f_{m}$ are the damping factor and resonant frequency, respectively. The set of $M$ poles is $\mathbf{s}_{M}=\left[s_{0}, s_{1}, \ldots, s_{M-1}\right]^{\mathrm{T}}$, where $[\cdot]^{\mathrm{T}}$ denotes the transpose.

In (1), time and frequency variation is represented by $N$ polynomials and $M$ pole terms, whereas spatial variation is described by $a_{n}(\mathbf{r})$ and $R_{m}(\mathbf{r})$. In general, $N$ and $M$ could be a function of $\mathbf{r}$; however, (1) is a more compact representation that uses a single set of $N$ polynomials and $M$ pole terms.

To successfully extrapolate a response one must determine $a_{n}(\mathbf{r}), R_{m}(\mathbf{r})$, and a set of poles $\mathbf{s}_{M}$, such that $\widehat{x}(\mathbf{r}, t)$ and $\widehat{X}(\mathbf{r}, f)$ in (1) accurately represent $x(\mathbf{r}, t)$ and $X(\mathbf{r}, f)$, respectively, for time and frequency points of interest. To do so, one first uses CEM methods to determine numerical values of $x(\mathbf{r}, t)$ and $X(\mathbf{r}, f)$ at discrete spatial positions in $\mathscr{R}$ for each early-time and low-frequency point, respectively.

Let $\mathbf{r}_{i}$ be a vector defining the $i$ th discrete spatial position in $\mathscr{R}$. The total number of positions in $\mathscr{R}$ is denoted by $I$, and the corresponding set of position vectors is $\mathscr{I}=$ $\left\{\mathbf{r}_{1}, \mathbf{r}_{2}, \ldots, \mathbf{r}_{i}, \ldots, \mathbf{r}_{I}\right\}$. Figure 1 illustrates an arbitrary region $\mathscr{R}$ and a set of discrete position vectors defined over the region. The discrete time-domain response at $\mathbf{r}_{i}$ is denoted by $\mathbf{x}^{i}$ and contains $P$ time samples of $x\left(\mathbf{r}_{i}, t\right)$ at $\Delta t$ intervals. The early-time data vector $\mathbf{x}_{\mathrm{ET}}^{i}$ contains the first $p$ samples of $\mathbf{x}^{i}$. Similarly, $\mathbf{X}^{i}$ is the discrete frequency-domain response at $\mathbf{r}_{i}$ which contains $Q$ samples of $X\left(\mathbf{r}_{i}, f\right)$ at $\Delta f$ intervals. The low-frequency data vector $\mathbf{X}_{\mathrm{LF}}^{i}$ contains the first $q$ samples of $\mathbf{X}^{i}[1,2]$.

First, CEM methods are used to determine $\mathbf{x}_{\mathrm{ET}}^{i}$ and $\mathbf{X}_{\mathrm{LF}}^{i}$ for the $I$ positions in $\mathscr{R}$. The response is extrapolated by obtaining $\mathbf{s}_{M}$ and then determining $\mathbf{a}_{N}\left(\mathbf{r}_{i}\right)$ and $\mathbf{R}_{M}\left(\mathbf{r}_{i}\right)$ for each $\mathbf{r}_{i}$. In this work, a multisignal formulation of MPM is used to determine $\mathbf{s}_{M}$ from early-time data.

After determining the poles, $\mathbf{a}_{N}\left(\mathbf{r}_{i}\right)$ and $\mathbf{R}_{M}\left(\mathbf{r}_{i}\right)$ are found by fitting $\mathbf{X}_{\mathrm{ET}}^{i}$ and $\mathbf{X}_{\mathrm{LF}}^{i}$ and solving a system of $(p+q)$ linear equations, at each $\mathbf{r}_{i}$, defined as

$$
\left[\begin{array}{ll}
\phi_{N}\left[t_{\mathrm{ET}}\right] & \mathbf{g}_{M}\left[t_{\mathrm{ET}}\right] \\
\boldsymbol{\Phi}_{N}\left[f_{\mathrm{LF}}\right] & \mathbf{G}_{M}\left[f_{\mathrm{LF}}\right]
\end{array}\right]\left[\begin{array}{l}
\mathbf{a}_{N}\left(\mathbf{r}_{i}\right) \\
\mathbf{R}_{M}\left(\mathbf{r}_{i}\right)
\end{array}\right]=\left[\begin{array}{l}
\mathbf{x}_{\mathrm{ET}}^{i} \\
\mathbf{X}_{\mathrm{LF}}^{i}
\end{array}\right]
$$


In (2), the polynomial coefficient and pole residue vectors are

$$
\begin{aligned}
\mathbf{a}_{N}\left(\mathbf{r}_{i}\right) & =\left[a_{0}\left(\mathbf{r}_{i}\right), a_{1}\left(\mathbf{r}_{i}\right), \ldots, a_{N-1}\left(\mathbf{r}_{i}\right)\right]^{\mathrm{T}}, \\
\mathbf{R}_{M}\left(\mathbf{r}_{i}\right) & =\left[R_{0}\left(\mathbf{r}_{i}\right), R_{1}\left(\mathbf{r}_{i}\right), \ldots, R_{M-1}\left(\mathbf{r}_{i}\right)\right]^{\mathrm{T}},
\end{aligned}
$$

and the matrices obtained by evaluating the polynomials and poles at early-time and low-frequency points are written as

$$
\begin{gathered}
\phi_{N}\left[t_{\mathrm{ET}}\right]=\left[\begin{array}{ccc}
\phi_{0}\left(\frac{t_{1}}{\ell_{1}}\right) & \cdots & \phi_{N-1}\left(\frac{t_{1}}{\ell_{1}}\right) \\
\vdots & \ddots & \vdots \\
\phi_{0}\left(\frac{t_{p}}{\ell_{1}}\right) & \cdots & \phi_{N-1}\left(\frac{t_{p}}{\ell_{1}}\right)
\end{array}\right], \\
\mathbf{g}_{M}\left[t_{\mathrm{ET}}\right]=\left[\begin{array}{ccc}
g_{0}\left(t_{1}\right) & \cdots & g_{M-1}\left(t_{1}\right) \\
\vdots & \ddots & \vdots \\
g_{0}\left(t_{p}\right) & \cdots & g_{M-1}\left(t_{p}\right)
\end{array}\right], \\
\boldsymbol{\Phi}_{N}\left[f_{\mathrm{LF}}\right]=\left[\begin{array}{ccc}
\Phi_{0}\left(\frac{f_{1}}{\ell_{2}}\right) & \cdots & \Phi_{N-1}\left(\frac{f_{1}}{\ell_{2}}\right) \\
\vdots & \ddots & \vdots \\
\Phi_{0}\left(\frac{f_{q}}{\ell_{2}}\right) & \cdots & \Phi_{N-1}\left(\frac{f_{q}}{\ell_{2}}\right)
\end{array}\right], \\
\mathbf{G}_{M}\left[f_{\mathrm{LF}}\right]=\left[\begin{array}{ccc}
G_{0}\left(f_{1}\right) & \cdots & G_{M-1}\left(f_{1}\right) \\
\vdots & \ddots & \vdots \\
G_{0}\left(f_{q}\right) & \cdots & G_{M-1}\left(f_{q}\right)
\end{array}\right] .
\end{gathered}
$$

For cases of interest $(p+q)>(N+M)$ and (2) is an overdetermined system for which a least-squares solution can be determined [10]. As a consequence of (1) the matrices in (4) are not a function of $\mathbf{r}_{i}$ and therefore need only be filled once when solving (2) for each of the $I$ spatial positions. The extrapolation is successful if $\widehat{x}(\mathbf{r}, t)$ and $\widehat{X}(\mathbf{r}, f)$ in (1) accurately represent the response, at each $\mathbf{r}_{i}$, in the directlycomputed early-time and low-frequency ranges, and in the extrapolated late-time and high-frequency ranges.

\section{Reliable Selection of Parameters}

A successful extrapolation depends on the proper selection of three pole-estimation parameters and three polynomial parameters needed to determine the poles $\mathbf{s}_{M}$, and coefficients $\mathbf{a}_{N}\left(\mathbf{r}_{i}\right)$ and residues $\mathbf{R}_{M}\left(\mathbf{r}_{i}\right)$ in (1). In this work all parameters are efficiently and reliably selected by fitting the response, in early time and low frequency, at a small number of locations in $\mathscr{R}$. The procedure is automated with a GA-based procedure as in $[1,2]$. In this work, however, the GA procedure is extended to fit the response simultaneously at multiple points in space in order to more efficiently extrapolate a response defined over a spatial region.

3.1. Extrapolation Parameters. Three polynomial parameters must be selected to successfully extrapolate a response: the number of polynomials in the expansion $N$, the time scaling factor $\ell_{1}$, and the time center $\tau[1,2]$. The selection of the time scaling factor $\ell_{1}$ defines the time and frequency support of the polynomials, which in this work are associate Hermite (AH) functions [9]. The value of $N$ specifies the number of polynomials used to represent the response, as in (1). Empirical stability bounds for $N$ and $\ell_{1}$ have been presented for several polynomial types [11]; however, there remains no precise criterion to select optimal values for a given response. Consequently, $N$ and $\ell_{1}$ are selected with the optimizationbased procedure in this section. Note that because $\ell_{1}$ and $\ell_{2}$ are directly related by $\ell_{2}=1 /\left(2 \pi \ell_{1}\right)$, selecting one parameter defines the other. Thus, only one scaling factor must be selected through optimization. The third polynomial parameter is the time center, $\tau$, which centers the polynomials in the time domain around $t=\tau$ rather than at $t=0$. This parameter is important to the extrapolation because associate Hermite functions in the time domain provide equal support around the origin, and it is assumed that the time-domain response is zero prior to $t=0$. In practice, the proper selection of $\tau$ can lead to a more efficient representation of the response (e.g., smaller $N$ ) and is critical to the numerical stability of the extrapolation. Typically, the optimal time center is around half of the time support of the response; however, no precise selection criteria exists, so $\tau$ is selected with the optimization procedure described in this section.

Parameters associated with the pole-estimation method must also be selected. In this work, poles are estimated from early-time data using a multisignal formulation of the matrix pencil method (MPM) described in [5]. MPM is efficient and generally numerically robust; however, in practice the accuracy of the procedure depends on the selection of the time samples processed. The data vector processed by the MPM algorithm is selected as a subset of the early-time data samples in $\mathbf{x}_{\mathrm{ET}}^{i}$. It can be uniquely specified with three positive integers: the beginning time sample $p_{1}$, the ending time sample $p_{2}$, and a decimation factor $d$. Note that $p_{1}<$ $p_{2}<p$, where $p$ is the total number of early-time points in $\mathbf{x}_{\mathrm{ET}}^{i}$.

The values of $p_{1}$ and $p_{2}$ define the first and last time samples in $\mathbf{x}_{\mathrm{ET}}^{i}$ to be processed with MPM. These parameters provide a means to identify an optimal time window within the available early-time data to estimate the poles. The decimation factor, $d$, is defined such that every $d$ th time sample in $\mathbf{x}_{\mathrm{ET}}^{i}$ is retained. The sampling time $\Delta t$ of the earlytime data is typically selected to satisfy stability requirements of the time-domain CEM method; however, this often results in oversampling compared to a favorable $\Delta t$ for MPM. Therefore, decimation of the time-domain data $\mathbf{x}_{\mathrm{ET}}^{i}$ is generally required to accurately estimate the poles.

The resulting data vector contains $\left\lfloor\left(p_{2}-p_{1}\right) / d\right\rfloor$ time samples uniformly spaced at $d \Delta t$ intervals, where $[\cdot\rfloor$ is the greatest integer less than or equal to the argument. Explicitly, the early-time data vector $\mathbf{x}_{\mathrm{ET}}^{i}$ contains samples of the response at time steps $\{\Delta t, 2 \Delta t, \ldots, p \Delta t\}$, whereas the time samples processed by MPM are defined at time steps $\left\{p_{1} \Delta t,\left(p_{1}+\right.\right.$ d) $\left.\Delta t,\left(p_{1}+2 d\right) \Delta t, \ldots, p_{2} \Delta t\right\}$. In practice, the success of the extrapolation depends on the proper selection of $\left\{p_{1}, p_{2}, d\right\}$, which allows one to determine $s_{M}$. Note that by poles estimating poles from early-time data, a time-domain bias 


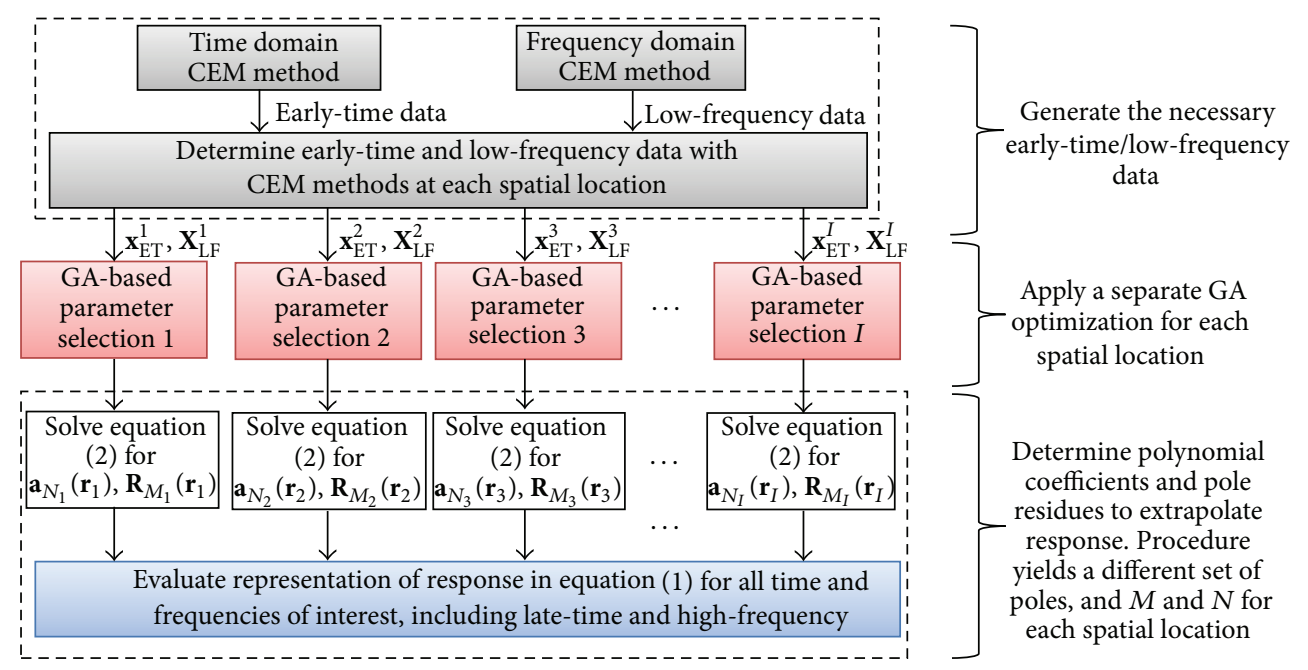

FIGURE 2: This flow chart illustrates how a spatial response can be extrapolated by repeatedly applying the procedure in [2] at each spatial location. This approach has two significant disadvantages: first, it requires a separate GA optimization at each spatial location, and second the process does not result in a single set of poles or constant number of polynomials to represent the spatial response.

is consequently introduced as discussed in [2]. Conversely, poles can also be estimated from low-frequency data instead using a frequency-domain technique such as the vector fitting method [12].

3.2. Parameter Selection. The GA-based procedure in [2] may be used to reliably extrapolate a response at a point in space, and a spatial response in $\mathscr{R}$ may be viewed as $I$ point responses at positions $\mathbf{r}_{i}$ for $i=1,2, \ldots, I$. Therefore, one could extrapolate the response over a spatial region by applying the procedure in [2] at each of the $I$ locations and fitting the response at each $\mathbf{r}_{i}$. This approach is illustrated in Figure 2. The computational expense of this approach, however, is prohibitive because $I$ optimizations are required, which even for moderately-sized regions can be large. Additionally, by selecting parameters at each $\mathbf{r}_{i}, N$ and $M$ of (1) are not necessarily constant over $\mathscr{R}$. For efficiency it is desired to represent the response with a single set of $N$ polynomials and $M$ pole terms.

Instead of selecting parameters by fitting the response at each $\mathbf{r}_{i}$, a second approach would be to select parameters by fitting the response at a single position $\mathbf{r}_{i}^{\prime}$ selected from $\mathscr{I}=$ $\left\{\mathbf{r}_{1}, \mathbf{r}_{2}, \ldots, \mathbf{r}_{I}\right\}$. The set of poles and polynomial parameters determined by fitting the response at $\mathbf{r}_{i}^{\prime}$ could then be used in (2) to determine $\mathbf{a}_{N}\left(\mathbf{r}_{i}\right)$ and $\mathbf{R}_{M}\left(\mathbf{r}_{i}\right)$ for the other $I-1$ positions. While this approach gives a constant $M$ and $N$, the parameters selected by fitting at only $\mathbf{r}_{i}^{\prime}$ may not (and does not in practice) provide a good fit at all other spatial positions, and $\mathbf{s}_{M}$ may not contain accurate estimates of all the poles.

A more efficient and reliable procedure to extrapolate a spatial response than the two described above is to select extrapolation parameters based on simultaneously fitting the response at a subset of the $I$ positions in $\mathscr{R}$. The subset of positions where the response is fit is denoted by $\mathscr{K}=\left\{\mathbf{r}_{1}^{\prime}, \mathbf{r}_{2}^{\prime}, \ldots, \mathbf{r}_{K}^{\prime}\right\}$, which contains $K$ positions randomly selected from $\mathscr{I}$, where one hopes that $K \ll I$. With this approach, one uses MPM to estimate a single set of poles by simultaneously processing early-time data at the $K$ positions in $\mathscr{K}$. To determine the coefficients and residues, the system of equations in (2), which fits the response at a single position, is modified to fit the response at $K$ positions (see Section 3.4). A GA is used to select parameters which yield an accurate representation of the response at each of the $K$ locations (see Section 3.3). After $\left\{p_{1}, p_{2}, d\right\}$ and $\left\{N, \ell_{1}, \tau\right\}$ are selected, MPM is used to determine $\mathbf{s}_{M}$, and $\mathbf{a}_{N}\left(\mathbf{r}_{i}\right)$ and $\mathbf{R}_{M}\left(\mathbf{r}_{i}\right)$ are found by solving (2) at each $\mathbf{r}_{i}$. The computational expense of solving (2) is minimal after the parameters have been selected.

Instead of selecting the $K$ fitting locations in a geometrically-regular arrangement, in this work the positions are randomly distributed in $\mathscr{R}$. The exact locations of the positions are not critical, but rather fitting at multiple locations should provide the diversity needed to accurately extrapolate the entire response. Selecting the $K$ positions in a random arrangement attempts to avoid fitting at positions corresponding to nulls of the response at each resonance. Thus, the random arrangement increases the likelihood that an accurate set of poles will be determined. For the numerical examples considered by the author, an acceptable value of $K$ ranged from 5 to 10 , which was significantly less than the total number of spatial locations. Increasing the value of $K$ did not improve noticeably accuracy but does increase the computational expense of applying the procedure. Therefore, a small value of $K$ is preferable.

3.3. GA Minimization. The accuracy of the extrapolation is a function of each parameter described in Section 3.1. Selecting the extrapolation parameters can be viewed as an optimization problem where the optimal set of parameters minimizes the difference between the directly-computed response and extrapolated representation in (1). The goal is to automate the parameter selection such that an arbitrary response can be reliably and accurately extrapolated. One must select the type of optimization used and a metric to quantity the "goodness" of a solution. GA optimization is 
used here because it is robust against local minimums and requires no a priori knowledge of the characteristics of the function being optimized.

To apply the GA one must define a fitness function which quantifies the "goodness" of a solution. The extrapolation agreement at $\mathbf{r}_{i}$, denoted $E_{i}$, can be quantified with a normalized mean square difference between the true response in time and frequency and the extrapolated representations $\widehat{x}(\mathbf{r}, t)$ and $\widehat{X}(\mathbf{r}, f)[1$, eq. (10)]. Thus, the goal is to select parameters which minimize $E_{i}$ at each $\mathbf{r}_{i}$. Doing so yields a representation of the response $\widehat{x}(\mathbf{r}, t)$ and $\widehat{X}(\mathbf{r}, f)$ as defined in (1) that accurately represents the true response for all time and frequency. Computing $E_{i}$, however, requires complete knowledge of the response, which will not be known at the time of the extrapolation. Therefore, this metric cannot be directly minimized in practice. In previous works by the authors, it is shown that an approximation of $E_{i}$, denoted $\widehat{E}_{i}$, can be minimized in place of $E_{i}$ to reliably select extrapolation parameters $\left[1\right.$, eq. (11)]. Computing $\widehat{E}_{i}$ requires only early-time and low-frequency data and therefore is useful in practical applications. Agreement in the late-time and high-frequency regions where no data is available to directly compare against is determined using the fast Fourier transform (FFT), as first suggested in [4].

In $[1,2]$ it is shown that a response at a spatial point $\mathbf{r}_{i}$ can be reliably extrapolated by using parameters found with GA optimization to minimize $\widehat{E}_{i}$. To efficiently extrapolate responses over a spatial region extrapolation agreement is simultaneously considered at $K$ spatial positions. A new metric, $\widehat{E}_{\mathscr{K}}$, is defined here as the mean of $\widehat{E}_{i}$ over the $K$ locations of $\mathscr{K}$, written as

$$
\widehat{E}_{\mathscr{K}}=\frac{1}{K} \sum_{i=1}^{K} \widehat{E}_{i}
$$

The optimization-based procedure is as follows. First, the extrapolation parameters in Section 3.1 are selected by minimizing $\widehat{E}_{\mathscr{K}}$ in (5) with a GA. The resulting parameters are then used to extrapolate the response at each spatial location by solving the system of equations in (2) for each $\mathbf{r}_{i}$ to determine $\mathbf{a}_{N}\left(\mathbf{r}_{i}\right)$ and $\mathbf{R}_{M}\left(\mathbf{r}_{i}\right)$.

\subsection{Fitting with a Small Subset of Spatial Positions. Extrapo-} lating a spatial response with the procedure described above requires that a single set of poles be determined by simultaneously processing early-time data at $K$ spatial locations. The standard MPM algorithm, however, determines the poles of a single signal [13]. In this work a multisignal variation of MPM, described in [5], is utilized to process data from $K$ positions and determine a single set of poles. In [5] the data processed corresponds to transient signals scattered by a structure and observed at different look angles. It is assumed the same set of poles is present in each signal and that the coefficient of each pole term is a function of observation angle. These assumptions are applied here to a spatial response. The poles are intrinsic to the structure, whereas the weighting of each pole term has spatial dependence as in (1).
The system of equations in (2) fits the response at a single location $\mathbf{r}_{i}$ but can be modified to fit the response at $K$ spatial locations, defined by $\mathscr{K}=\left\{\mathbf{r}_{1}^{\prime}, \mathbf{r}_{2}^{\prime}, \ldots, \mathbf{r}_{K}^{\prime}\right\}$, as

$$
\left[\begin{array}{cc}
\boldsymbol{\phi}_{N}\left[t_{\mathrm{ET}}\right] & \mathbf{g}_{M}\left[t_{\mathrm{ET}}\right] \\
\boldsymbol{\Phi}_{N}\left[f_{\mathrm{LF}}\right] & \mathbf{G}_{M}\left[f_{\mathrm{LF}}\right]
\end{array}\right]\left[\begin{array}{c}
\mathbf{a}_{N}[\mathscr{K}] \\
\mathbf{R}_{M}[\mathscr{K}]
\end{array}\right]=\left[\begin{array}{c}
\mathbf{x}_{\mathrm{ET}}^{\mathscr{K}} \\
\mathbf{X}_{\mathrm{LF}}^{\mathscr{K}}
\end{array}\right]
$$

In (6), $\mathbf{a}_{N}[\mathscr{K}]$ is a $(N \times K)$ matrix containing polynomials coefficients, defined as

$$
\mathbf{a}_{N}[\mathscr{K}]=\left[\begin{array}{ccc}
a_{0}\left(\mathbf{r}_{1}^{\prime}\right) & \cdots & a_{0}\left(\mathbf{r}_{K}^{\prime}\right) \\
\vdots & \ddots & \vdots \\
a_{N-1}\left(\mathbf{r}_{1}^{\prime}\right) & \cdots & a_{N-1}\left(\mathbf{r}_{K}^{\prime}\right)
\end{array}\right]
$$

and $\mathbf{R}_{M}[\mathscr{K}]$ is a $(M \times K)$ matrix containing pole residues, defined as

$$
\mathbf{R}_{M}[\mathscr{K}]=\left[\begin{array}{ccc}
R_{0}\left(\mathbf{r}_{1}^{\prime}\right) & \cdots & R_{0}\left(\mathbf{r}_{K}^{\prime}\right) \\
\vdots & \ddots & \vdots \\
R_{M-1}\left(\mathbf{r}_{1}^{\prime}\right) & \cdots & R_{M-1}\left(\mathbf{r}_{K}^{\prime}\right)
\end{array}\right] .
$$

The data vectors on the right-hand-side of (6) contain earlytime and low-frequency CEM samples as

$$
\begin{gathered}
\mathbf{x}_{\mathrm{ET}}^{\mathscr{K}}=\left[\begin{array}{ccc}
x\left(\mathbf{r}_{1}^{\prime}, t_{1}\right) & \cdots & x\left(\mathbf{r}_{K}^{\prime}, t_{1}\right) \\
\vdots & \ddots & \vdots \\
x\left(\mathbf{r}_{1}^{\prime}, t_{p}\right) & \cdots & x\left(\mathbf{r}_{K}^{\prime}, t_{p}\right)
\end{array}\right], \\
\mathbf{X}_{\mathrm{LF}}^{\mathscr{K}}=\left[\begin{array}{ccc}
X\left(\mathbf{r}_{1}^{\prime}, f_{1}\right) & \cdots & X\left(\mathbf{r}_{K}^{\prime}, f_{1}\right) \\
\vdots & \ddots & \vdots \\
X\left(\mathbf{r}_{1}^{\prime}, f_{q}\right) & \cdots & X\left(\mathbf{r}_{K}^{\prime}, f_{q}\right)
\end{array}\right] .
\end{gathered}
$$

The matrices in (4) appear in (6) and are unchanged by the extension of (2) to fit at $K$ positions. It is preferable to select a small $K$ because the computational expense of solving (6) is proportional to $K$, as (7)-(9) each has $K$ columns. The system of equations in (6), combined with MPM applied to multiple data sets, allows one to select the necessary parameters with a GA by fitting the response at $K$ positions and minimizing $\widehat{E}_{\mathscr{K}}$ in (5). The selection of $\left\{p_{1}, p_{2}, d\right\}$ allows an accurate set of poles $\mathbf{s}_{M}$ to be determined with MPM, and $\left\{N, \ell_{1}, \tau\right\}$ are used when solving (2) at each $\mathbf{r}_{i}$ in $\mathscr{R}$.

Figure 2 illustrates the process by which one could repeated apply the procedure in [2] to extrapolate a spatial response. However this approach is highly-inefficient, as a separate GA optimization is required to extrapolate the response at each spatial location. The GA optimization is by far the largest contributor to the CPU time when extrapolating the response. Conversely, Figure 3 illustrates the procedure described in this section to efficiently extrapolate a spatial response. Note that only one GA optimization is required and that the process results in a single set of poles and polynomial terms. This representation of the response is compact and can provide insight into structural resonances. 


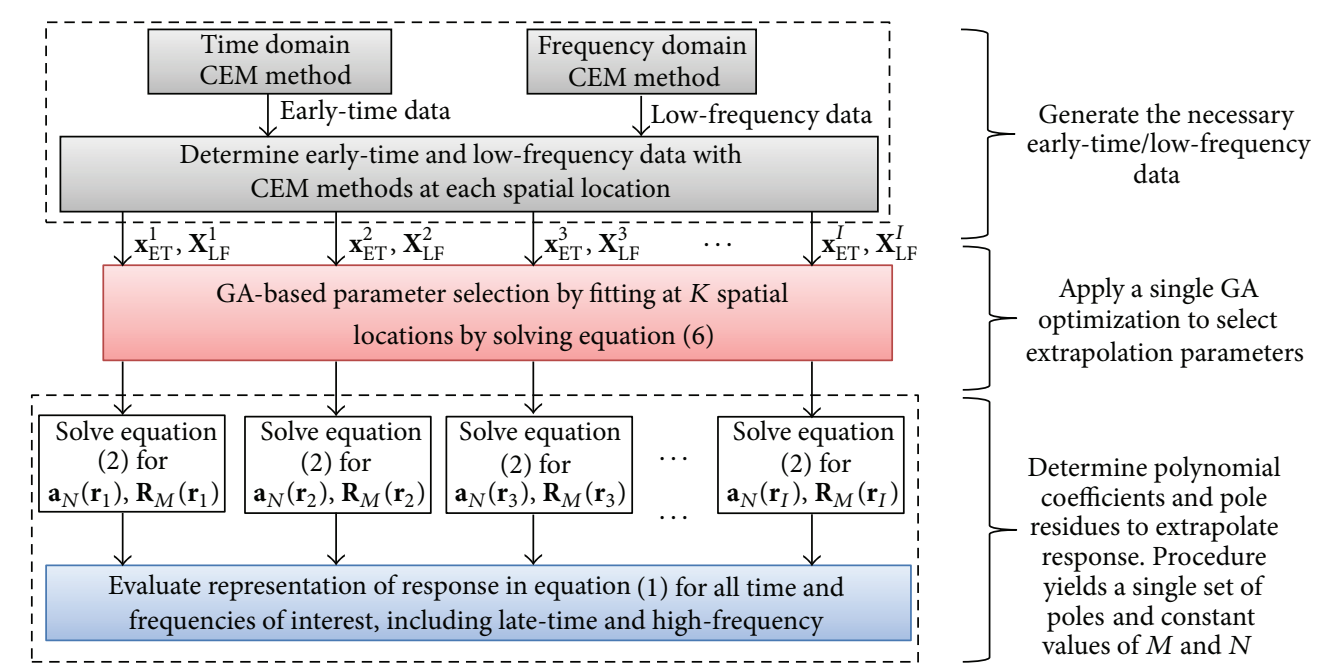

FIgure 3: This flow chart illustrates the procedure in this work to efficiently extrapolate a spatial response. Only one GA optimization is required, which significantly reduces computational time. Also, the procedure yields a representation of the spatial response as a single set of poles and constant number of polynomials.

\section{Determining Physical Poles}

Poles estimated from numerical data using techniques such as MPM can be classified as physical poles, corresponding to structural resonances, and fitting poles, which are not associated with resonances but can improve fit accuracy. It is desirable to identify physical poles when representing a response as in (1), but distinguishing them from fitting poles is often difficult when the resonant frequencies are unknown.

Various procedures are presented in the literature to distinguish between physical and fitting poles, such as those in [6-8]. However, the automated application of such procedures is practice is often difficult. A new automated procedure is presented here to distinguish between physical and fitting poles. First, one uses MPM to estimate poles at $K$ spatial locations randomly distributed in $\mathscr{R}$ as described in Section 3. Three sets of poles are determined by fitting the response at different sets of $K$ position vectors denoted $\mathscr{K}_{1}, \mathscr{K}_{2}$, and $\mathscr{K}_{3}$, respectively. The resulting sets of poles are compared, and the poles coincident in all three sets are assumed to be the physical poles, whereas noncoincident poles are classified as the fitting poles. After identifying the physical poles a response can be represented, as in (1), with each of the $M$ pole terms corresponding to a physical pole. To determine a representation with polynomials and physical poles only, fitting poles are removed from $\mathbf{s}_{M}$; then coefficients and residues are determined by solving (2) for each $\mathbf{r}_{i}$.

This approach is motivated by the fact that physical poles are intrinsic to the characteristics of the structure or system, and therefore only a single set of poles is needed to represent a spatial response. For instance, the length of a dipole antenna defines its resonant frequencies. The locations of fitting poles, on the other hand, are not unique. Consequently, as the set of fitting locations is changed, the fitting poles change but the physical poles do not. This observation is used to distinguish between physical and fitting poles.

The spatial residues of physical poles, referred to here as modal residues, provide insight into the resonant behavior of a structure, whereas the spatial residues of fitting poles do not. Modal residues have spatial behavior similar to the natural modes determined by the singularity expansion method (SEM) [14]. The set of modal residues can be interpreted as a decomposition of a response into the characteristic spatial behavior of each resonance. This perspective is potentially useful in applications where the effects of certain resonances must be suppressed or enhanced, for example, with excitation selection or by changing physical dimensions.

Modal residues depend on the EM quantity of the response, as well as the spatial region where the response is defined. Additionally, to determine a physical pole from a given response, the corresponding resonance must be excited by the excitation and/or feeding arrangement. Additionally, the excitation bandwidth determines the range of frequencies for which poles can be resolved.

\section{Results and Discussion}

5.1. Example 1. The first spatial response considered is the axial current of a thin-wire cylindrical dipole antenna, aligned along the $z$ axis, with length $L=0.5 \mathrm{~m}$ and radius $a=2.5 \mathrm{~cm}$. The spatial region $\mathscr{R}$ is specified by $z \in[0, L]$ and contains $I=99$ spatial locations equally-spaced along the length of the dipole. The dipole is driven at its center with a differentiated Gaussian pulse (DGP) voltage excitation, with effective frequency content up to $f_{\max }=3 \mathrm{GHz}$ [1]. The electric field integral equation is solved with MoM [15] to determine the frequency-domain current at the 99 locations. The response is sampled at $\Delta f=3 \mathrm{MHz}$ intervals up to $3 \mathrm{GHz}$. The time-domain response, which is sampled at $\Delta t=$ 8 ps up to $35 \mathrm{~ns}$, is obtained by taking the discrete Fourier transform (DFT) of the IE/MoM data. A time-domain CEM method could be used, but this approach removes discretization or modeling differences, which is convenient for the present purpose.

The current is successfully extrapolated using $15 \mathrm{~ns}$ of early-time data and $1.5 \mathrm{GHz}$ of low-frequency data. A GA 

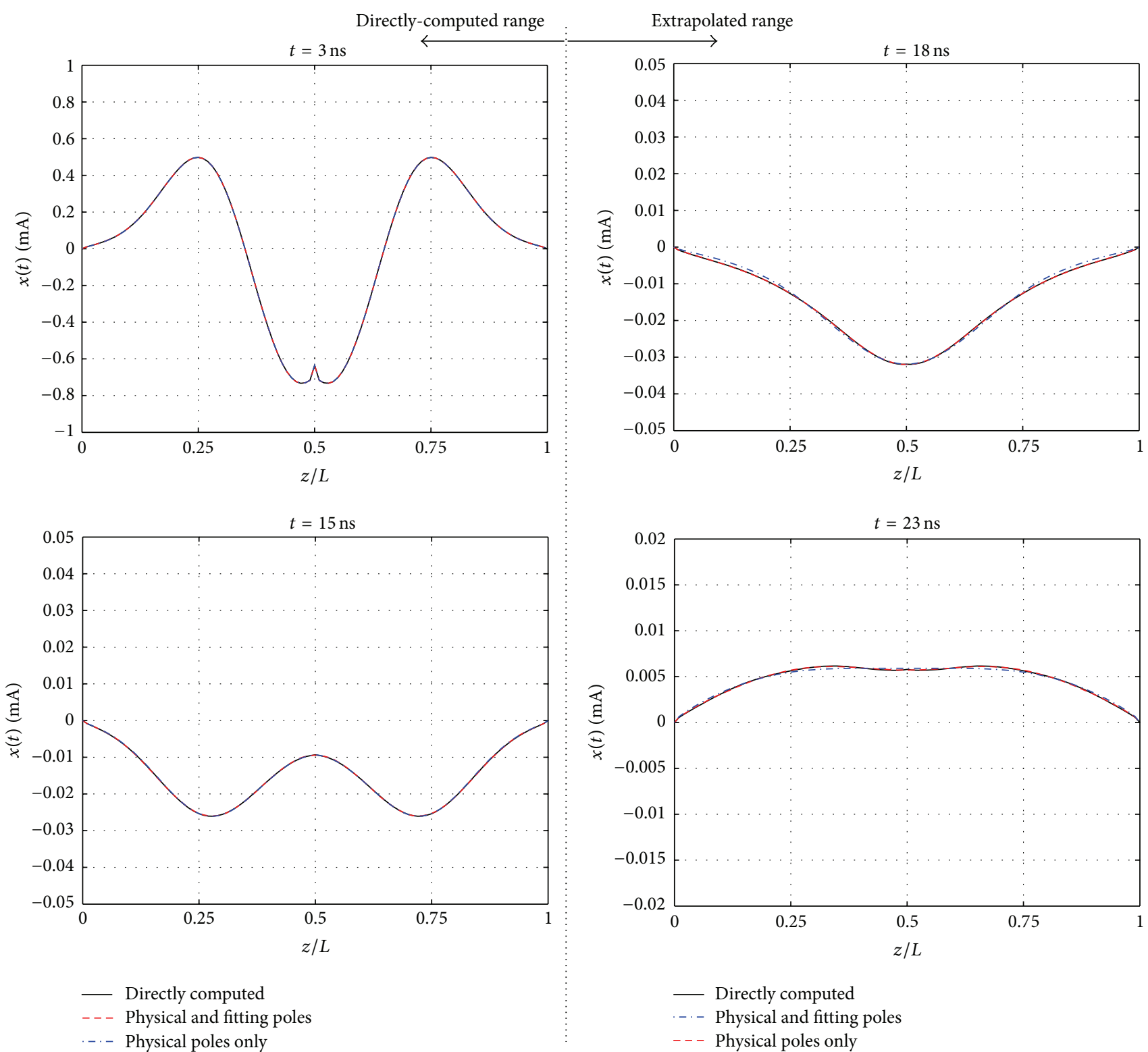

FIGURE 4: Axial current of center-fed dipole for several values of time. Directly-computed response, representation with polynomials, physical, and fitting pole terms, and representation with polynomials and physical pole terms only are indistinguishable, indicating a successful extrapolation $(K=5)$.

is used to select parameters that minimize $\widehat{E}_{\mathscr{K}}$ by fitting the response at $K=5$ positions randomly selected from the total of 99 . The exact locations of the 5 fitting positions are found not to be critical to the success of the extrapolation, but that simultaneously fitting at $K$ locations provides the diversity needed to select the necessary parameters.

Figure 4 compares the current determined with IE/MoM (directly computed) and two extrapolated representations of the spatial responses $(\widehat{x}(\mathbf{r}, t)$ and $\widehat{X}(\mathbf{r}, f)$ in (1)) at several values of time. The close agreement among the three curves for the points shown is typical of all time and frequencies of interest. The first extrapolated response (physical and fitting poles) is represented as in (1) with polynomials and both physical and fitting pole terms, while the second includes polynomials and only physical pole terms. Agreement among the three curves is excellent, indicating polynomials can provide the support for response behavior otherwise represented by fitting poles.

Figure 5 illustrates the poles $\left(s_{m}=\alpha_{m}+j 2 \pi f_{m}\right)$ of the response for three successful extrapolations. Parameters are selected by fitting the response at three different sets of 5 randomly selected locations, denoted by $\mathscr{K}_{1}, \mathscr{K}_{2}$, and $\mathscr{K}_{3}$. In Figure 5 the five poles coincident in the three pole sets are the physical poles, whereas the noncoincident poles are the fitting poles. The physical poles correspond to dipole resonances excited by the excitation and feeding arrangement. Higherorder resonances may be determined by increasing $f_{\max }$. The resonances occur when the electrical length of the dipole is approximately equal to an odd multiple of a half-wavelength; that is, $L \approx[(2 m-1) \lambda] / 2$ for $m=1,2,3,4,5$. The five physical 


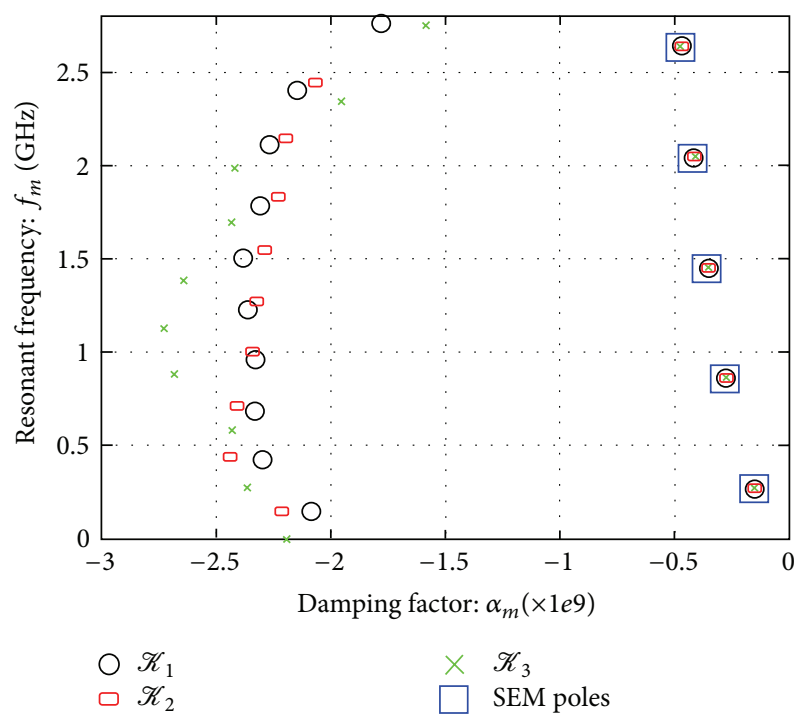

Figure 5: Poles of the dipole determined by fitting the response at $K=5$ spatial locations using three different sets of $K$ position vectors denoted by $\mathscr{K}_{1}, \mathscr{K}_{2}$, and $\mathscr{K}_{3}$. Physical poles are coincident in the three sets of poles, whereas fitting poles are not. SEM poles of the dipole are shown for comparison [14].

poles are used with polynomials to represent the response (physical poles only) in Figure 4.

The SEM poles, which correspond to the complex natural resonances of a structure [14], also provide a reference. In Figure 5 the physical poles determined by extrapolating IE/MoM data are shown to closely agree with the SEM poles in [14]. The average relative difference for resonant frequencies is $0.23 \%$ and for damping factors is $1.15 \%$. The small disagreement can be attributed in part to differences between the spatial discretization used in [14] and that in this work.

Figure 6 illustrates the modal residues of the dipole $R_{m}(z)$ which are determined by extrapolating the CEM data. The natural resonant behavior is apparent as the lowest-order residue $(m=1)$ resembles a half-wavelength sinusoid and high-order modal residues contain $[(2 m-1) \lambda] / 2$ wavelengths. Figure 6 can be interpreted as a decomposition of the current into modal functions, each associated with a structural resonance. Thus, the dipole current can be thought of as a superposition of the set of physical pole terms weighted by modal residues.

Figure 7 illustrates the value of extrapolation agreement $E_{i}$ determined at each of the $I=99$ positions on the dipole. The value of $E_{i}$ quantifies the agreement between the directlycomputed response and an extrapolated representation, where $E_{i}<10^{-2}$ is desired [2]. The two dashed/red curves in Figure 7 correspond to the two extrapolated responses shown in Figure 4, which are determined with a single GA optimization run to select parameters by fitting simultaneously at 5 locations. Conversely, for the two solid/black curves in Figure 7, the response is extrapolated with the procedure of [2] by applying a separate GA optimization run at each of the 99 spatial locations. This approach effectively provides a lower

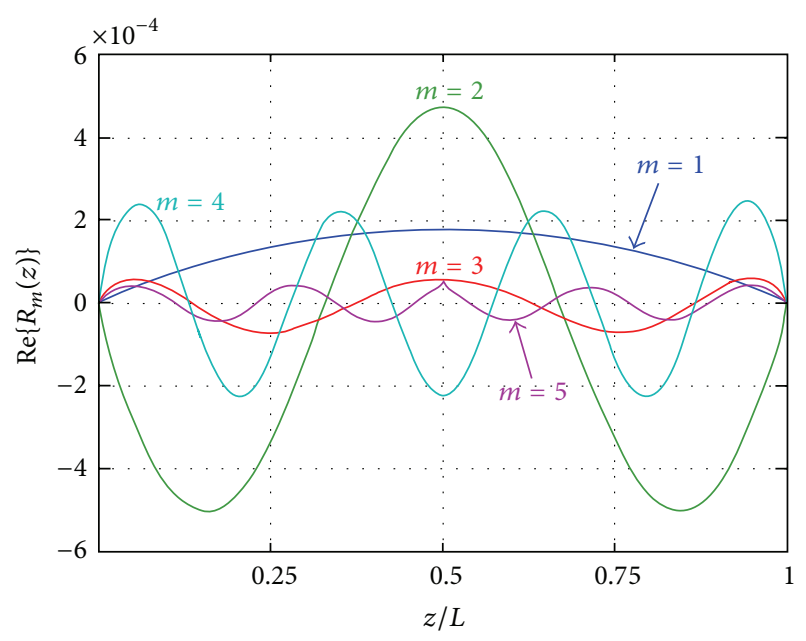

(a)

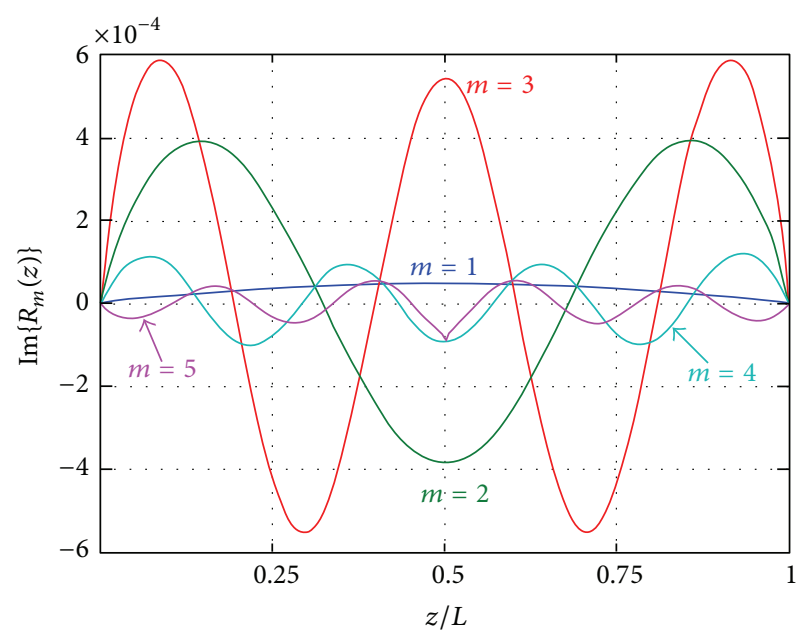

(b)

FIgURE 6: The modal residues $R_{m}(z)$ associated with the physical poles of a center-fed dipole: (a) real part, (b) imaginary part. Residues are determined by extrapolating CEM early-time and lowfrequency data and representing the dipole current as in (1).

bound on the value of $E_{i}$. Figure 7 shows only small gains in extrapolation agreement can be obtained with $K>5$, because $K=5$ closely approaches the lower bound. Therefore, the response is accurately extrapolated with $K \ll I$ as desired.

Extrapolating the response using the approach in this work requires a single optimization run, whereas applying the procedure of [2] requires 99 optimization runs. Reducing the number of optimizations required yields significant computational savings. To illustrate the computational differences, the procedure [2] was applied to extrapolate the response at each of the 99 positions in $\mathscr{R}$. Completing the 99 GA optimization runs required $900 \mathrm{~min}$ of total CPU time to extrapolate the response, whereas applying the procedure in this work required only $15.1 \mathrm{~min}$. Thus, the procedure in this work provides a $60 \mathrm{X}$ speed up relative to previous techniques.

Figure 7 also compares agreement between representations with polynomials and both physical and fitting poles 


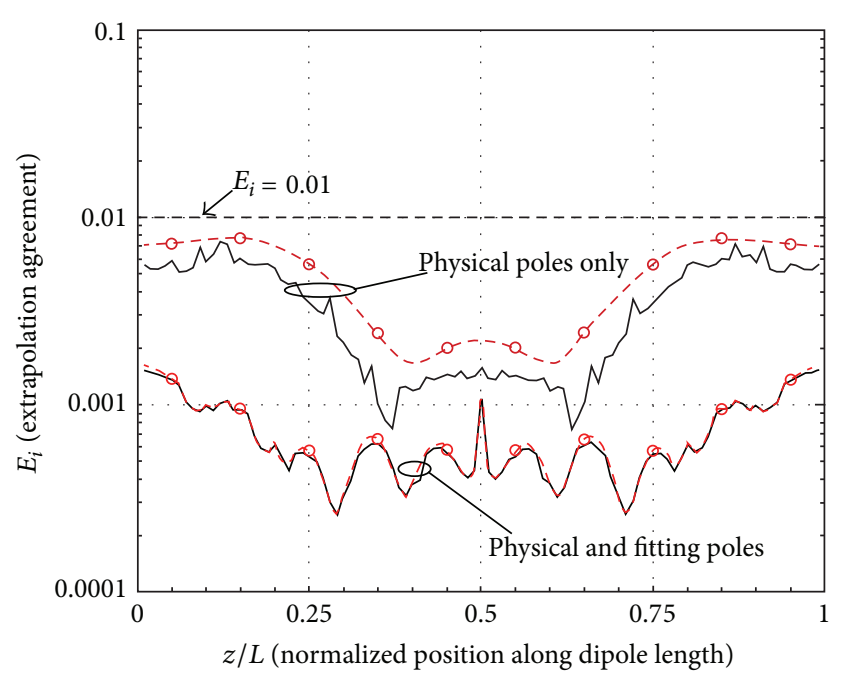

- Different optimization to fit each of the $I=99$ spatial locations (procedure outlined in Figure 2)

- $\ominus$ - Single optimization to fit at $K=5$ spatial locations (procedure outlined in Figure 3)

FIgURE 7: Comparison of the extrapolation agreement $E_{i}$ for the axial current of a center-fed dipole.

and representations with polynomials and only physical poles. The value of $E_{i}$ for the representations with polynomials and both physical and fitting poles is the lowest; however, the extrapolation agreement of the representation with polynomials and only physical poles is still $E_{i}<10^{-2}$. Figure 4 shows the representation with polynomials and only physical poles is nearly indistinguishable from the directlycomputed response, and therefore the response of the dipole is still accurately represented as polynomials and physical poles only. A representation of a response with only physical poles can be valuable in providing insight into the resonant behavior, but for best accuracy one may choose to use fitting poles in any extrapolation even with full knowledge of the physical poles.

To consider the effects of CEM modeling differences, the dipole response was determined in early time using a FDTD simulation and in low frequency using IE/MoM. The response was successfully extrapolated, despite discretization differences, using FDTD data up to $15 \mathrm{~ns}$ and IE/MoM data up to $1.5 \mathrm{GHz}$. The agreement between SEM poles and those determined via extrapolation is very good with an average relative difference of $1.54 \%$ for resonant frequencies and $1.51 \%$ for damping factors. The agreement is limited as expected by discretization differences between the FDTD and IE/MoM models $[1,2]$.

5.2. Example 2. The second resonant structure considered is the rectangular patch antenna in Figure 8. A DGP voltage excitation with $f_{\max }=7 \mathrm{GHz}$ is applied at the base of a probe feed connecting the ground plane and patch. The spatial response considered is the electric field $E_{z}$ in a $6 \mathrm{~cm} \times 8 \mathrm{~cm}$ region in the $x y$-plane between the patch and ground plane defined by $z=h / 2, x \in[-3 \mathrm{~cm}, 3 \mathrm{~cm}]$, and $y \in[-4 \mathrm{~cm}, 4 \mathrm{~cm}]$

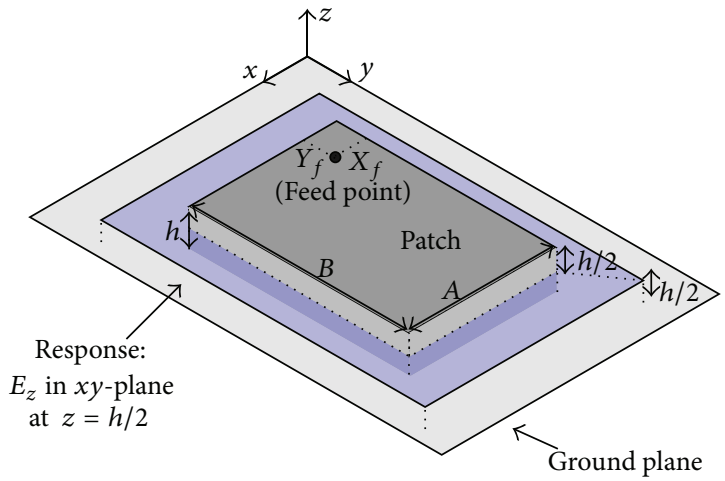

FIgURE 8: Geometry of rectangular patch antenna $(A=4 \mathrm{~cm}, B=$ $\left.6 \mathrm{~cm}, h=0.5 \mathrm{~cm}, X_{f}=1 \mathrm{~cm}, Y_{f}=0.8 \mathrm{~cm}\right)$. The ground plane is $8 \mathrm{~cm} \times 12 \mathrm{~cm}$ and the $E_{z}$ response region is $6 \mathrm{~cm} \times 8 \mathrm{~cm}$.

(Figure 8). The region $\mathscr{R}$ is discretized into cells with $\Delta x=$ $\Delta y=2 \mathrm{~mm}$, resulting in 30 and 40 locations along the $x$ and $y$ directions, respectively, for a total of $I=1,200$ locations in $\mathscr{R}$. Commercial FDTD software [16] is used to model the patch antenna and determine the time-domain response at each of the 1,200 spatial positions. The electric field is sampled at $\Delta t=54.2 \mathrm{ps}$ intervals up to $73.8 \mathrm{~ns}$. The frequency-domain response, which is sampled at $\Delta f=7 \mathrm{MHz}$ intervals up to $7 \mathrm{GHz}$, is determined as the DFT of the time response.

The spatial response of the patch antenna is successfully extrapolated using $10 \mathrm{~ns}$ of early-time data and $2.5 \mathrm{GHz}$ of low-frequency data. All parameters are selected with a GA to minimize $\widehat{E}_{K}$ by fitting at 10 spatial locations randomly selected from the 1,200 positions in $\mathscr{R}$. Figure 9 compares, at two frequency points, the directly-computed response with an extrapolated representation as in (1) using polynomials and physical pole terms only. The agreement at the frequency points shown is excellent and typical of all time and frequency points of interest. The value of $I$ for this example is significantly higher than the dipole response, and thus 10 fitting locations is used instead of 5. Extrapolating the response by fitting at $K>10$ positions does not result in a more accurate representation, and therefore fitting at $K \ll I$ locations provides the diversity to successfully extrapolate this response. It was again found that the specific choice of the 10 fitting locations is not critical to the success of the extrapolation.

To illustrate the efficiency of the procedure in this work, the patch response was also extrapolated by applying the procedure of [2] at each of the 1,200 positions in $\mathscr{R}$. The average CPU time required to extrapolate the response at each location was $8.6 \mathrm{~min}$, with a total of 172 hours needed to extrapolate response at all of the 1,200 positions in $\mathscr{R}$. Alternatively, extrapolating the spatial response with the procedure in this work required only $17 \mathrm{~min}$. The dramatic time savings is due to the fact that despite the large number of positions in $\mathscr{R}$, the approach in this work still only requires a single GA optimization to extrapolate the response.

Figure 10 illustrates the sets of poles determined by extrapolating the response of the patch antenna by selecting 

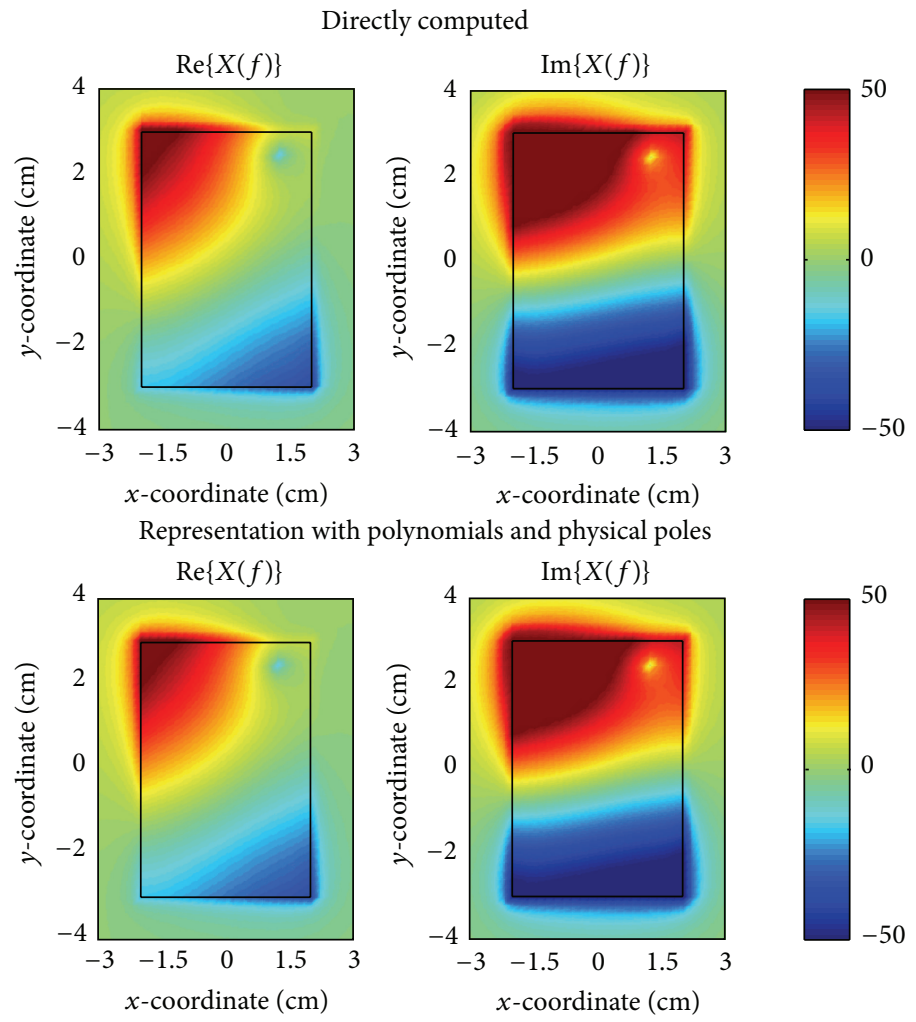

(a) $f=2.41 \mathrm{GHz}$

Directly-computed range

Extrapolated range

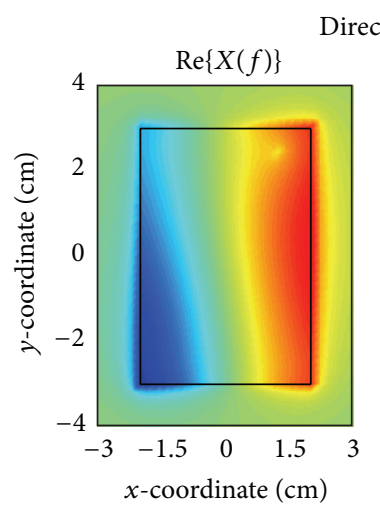

Directly computed

Representation with polynomials and physical poles
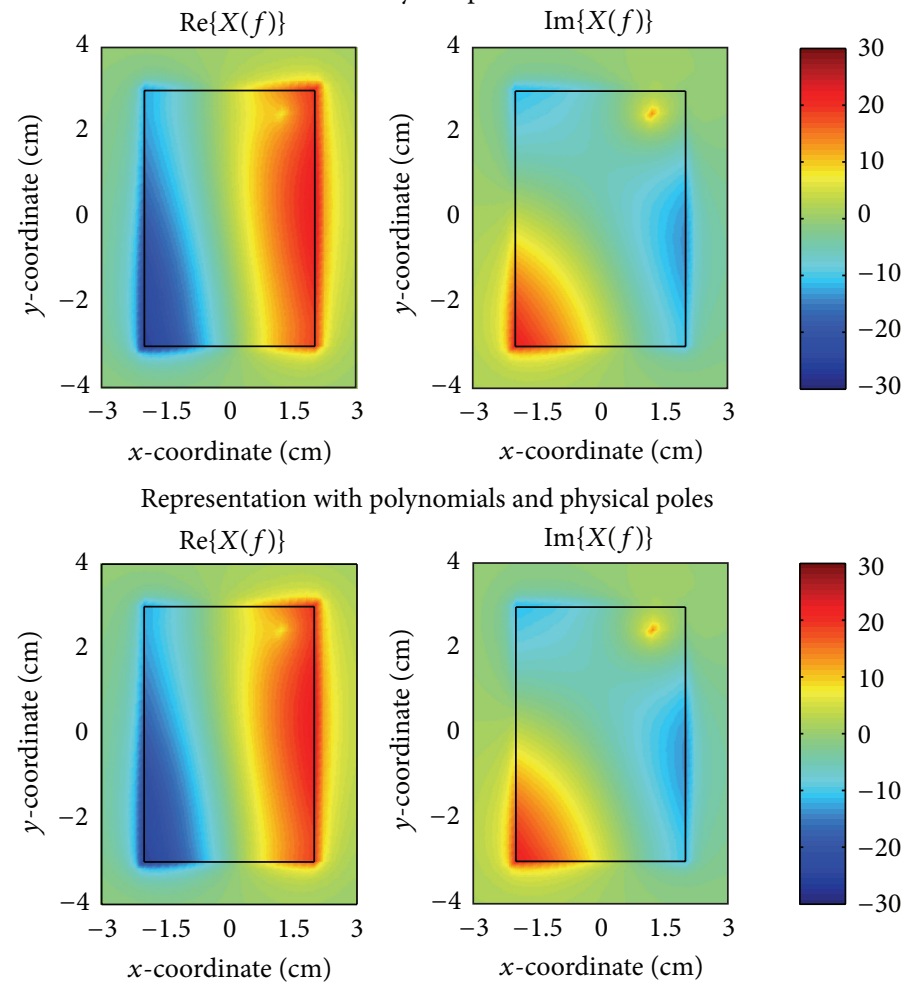

(b) $f=3.14 \mathrm{GHz}$

FIGURE 9: The electric field $E_{z}$ response of the patch antenna for (a) $f=2.41 \mathrm{GHz}$ and (b) $f=3.14 \mathrm{GHz}$. The directly-computed response and a representation with polynomials and physical poles only are nearly indistinguishable indicating a successful extrapolation $(K=10)$. 


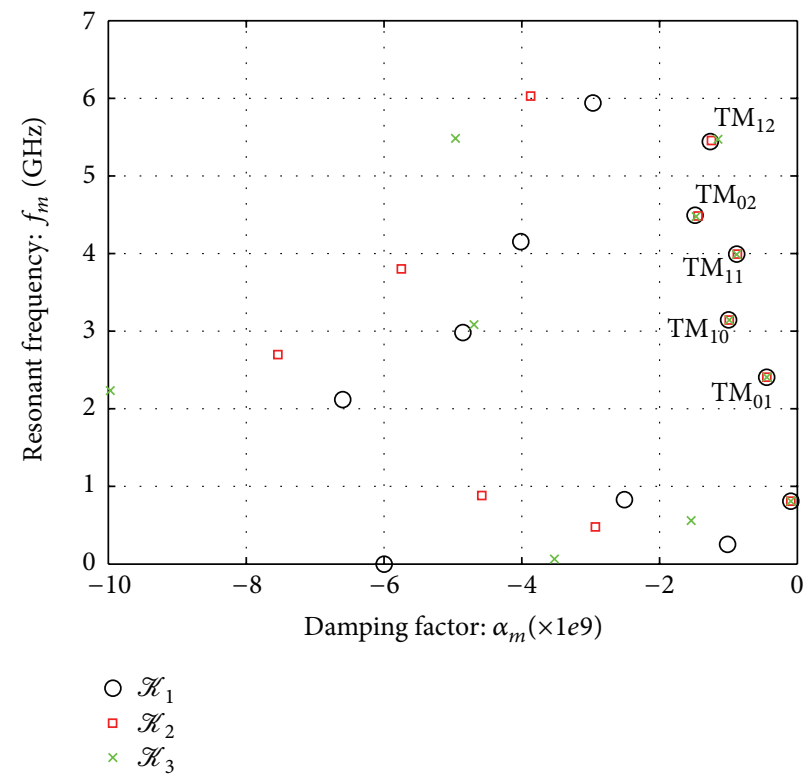

Figure 10: Poles of the patch antenna determined by fitting the response at $K=10$ spatial locations using three different sets of $K$ position vectors denoted by $\mathscr{K}_{1}, \mathscr{K}_{2}$, and $\mathscr{K}_{3}$. Physical poles are coincident in the three sets of poles, whereas the fitting pole is not.
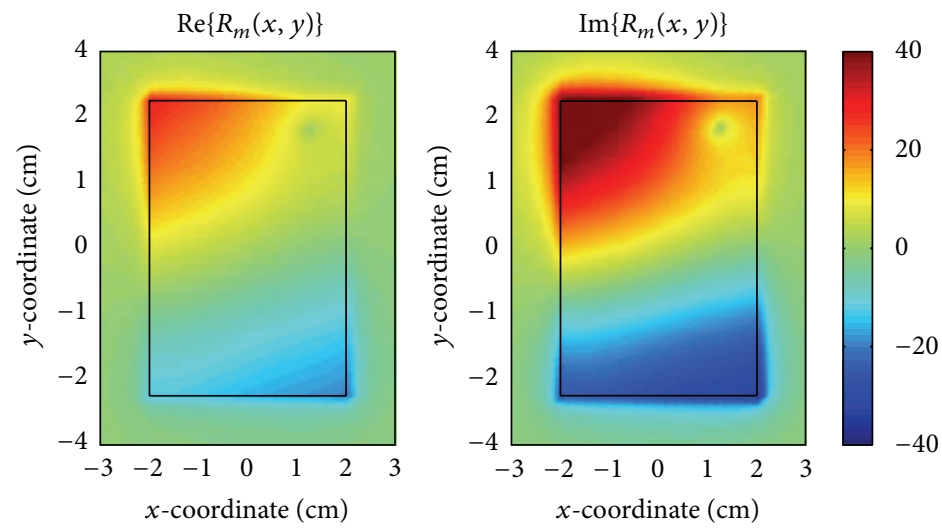

(a) $\mathrm{TM}_{01}\left(f_{m}=2.41 \mathrm{GHz}\right)$
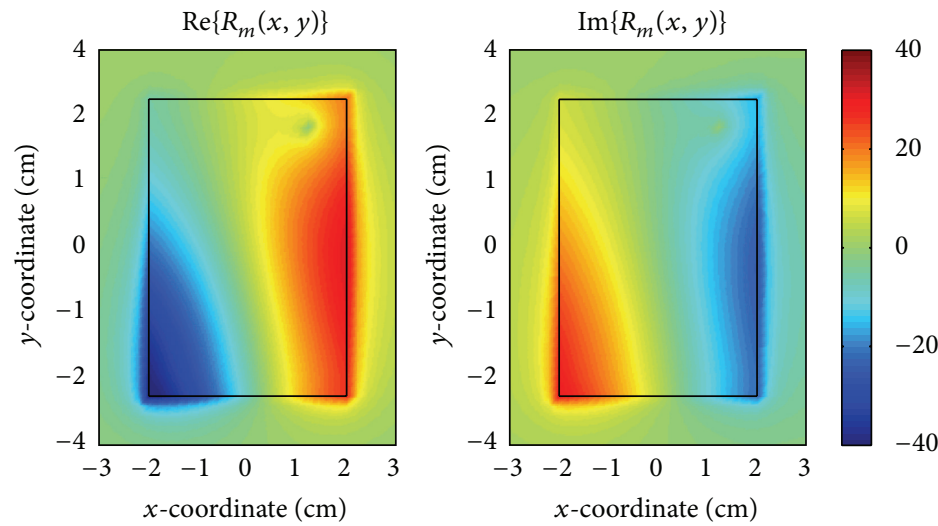

(b) $\mathrm{TM}_{10}\left(f_{m}=3.14 \mathrm{GHz}\right)$

FIgURE 11: The modal residue (see equations (2), (3) and (6)) for $\mathrm{TM}_{01}$ in (a) dominates the response shown in Figure 9(a). Similarly, the modal residues associated with the $\mathrm{TM}_{10}$ cavity mode in (b) dominates the response shown in Figure 9(b). 
parameters based on fitting at three different sets of 10 randomly-distributed positions, denoted by $\mathscr{K}_{1}, \mathscr{K}_{2}$, and $\mathscr{K}_{3}$. Physical poles are coincident in the three sets of poles, whereas the fitting poles are noncoincident. Each physical pole corresponds to a resonance due to a mode of the cavity formed by the patch and ground plane [17]. Because the height is small relative to the patch dimension, the dominate modes are mostly transverse magnetic to $z$. The antenna radiates effectively at frequencies near these resonances, and thus their determination is valuable.

Figure 11 shows the modal residues $R_{m}(x, y)$ which correspond to cavity modes $\mathrm{TM}_{01}$ and $\mathrm{TM}_{10}$. The spatial variation of the modal residues is very similar to the frequency-domain response at these resonant frequencies. It is also similar to the spatial variation of the corresponding cavity mode but is perturbed by the probe feed. A response can be viewed as a superposition of physical pole terms weighted by modal residue functions. With plots like Figure 11 the fundamental spatial behavior of each resonance can be examined individually. While the rectangular patch is relatively wellunderstood, this approach can also be used to study other antennas and/or complex systems with unknown resonant behavior.

\section{Conclusions}

A reliable and computationally efficient procedure is presented to extrapolate an EM response defined in an arbitrary spatial region. Previously, procedures efficient for responses at a single spatial location have been considered [1-4]. In this work an automated procedure is presented to extrapolate the spatial response with a single GA optimization run while selecting all needed parameters. It is shown that the spatial variation of the response can be represented with spatiallydependent polynomial coefficients and pole residues, and that a single set of poles is sufficient to describe the resonant behavior of the spatial response. A new automated procedure is also presented to distinguish the physical poles of a response from the fitting poles. With numerical examples, the physical poles of a dipole are shown to agree well with SEM poles and the physical poles of a patch antenna are shown to correspond to cavity modes. It is observed that the modal residues of physical poles can provide insight into the spatial behavior of structural resonances.

\section{Acknowledgment}

This work supported in part the Center for Research in Wireless Communication (CRWC) at Clemson University.

\section{References}

[1] J. M. Frye and A. Q. Martin, "Extrapolation of time and frequency responses of resonant antennas using damped sinusoids and orthogonal polynomials," IEEE Transactions on Antennas and Propagation, vol. 56, no. 4, pp. 933-943, 2008.

[2] J. M. Frye and A. Q. Martin, "Time and frequency bias in extrapolating wideband responses of resonant structures," IEEE Tra- nsactions on Antennas and Propagation, vol. 57, no. 12, pp. 39343941, 2009.

[3] M. M. Rao, T. Sarkar, T. Anjali, and R. Adve, "Simultaneous extrapolation in time and frequency domains using hermite expansions," IEEE Transactions on Antennas and Propagation, vol. 47, no. 6, pp. 1108-1115, 1999.

[4] M. Yuan, J. Koh, T. K. Sarkar, W. Lee, and M. Salazar-Palma, "A comparison of performance of three orthogonal polynomials in extraction of wide-band response using early time and low frequency data," IEEE Transactions on Antennas and Propagation, vol. 53, no. 2, pp. 785-792, 2005.

[5] T. K. Sarkar, S. Park, J. Koh, and S. M. Rao, "Application of the matrix pencil method for estimating the SEM (Singularity Expansion Method) poles of source-free transient responses from multiple look directions," IEEE Transactions on Antennas and Propagation, vol. 48, no. 4, pp. 612-618, 2000.

[6] J. F. Callejön, A. R. Bretones, and R. G. Martin, “On the application of parametric models to the transient analysis of resonant and multiband antennas," IEEE Transactions on Antennas and Propagation, vol. 46, no. 3, pp. 312-317, 1998.

[7] A. Driouach, A. R. Bretones, and R. G. Martin, "Application of parametric models to inverse scattering problems," IEE Proceedings Microwaves, Antennas and Propagation, vol. 143, no. $1,1996$.

[8] N. Fichtner, U. Siart, Y. Kuznetsov, A. Baev, and P. Russer, “TLM modeling and system identification of optimized antenna structures," Advances in Radio Science, vol. 6, pp. 45-48, 2008.

[9] L. R. Lo Conte, R. Merletti, and G. V. Sandri, "Hermite expansions of compact support waveforms: applications to myoelectric signals," IEEE Transactions on Biomedical Engineering, vol. 41, no. 12, pp. 1147-1159, 1994.

[10] G. H. Golub and C. F. V. Loan, Matrix Computations, Johns Hopkins University Press, Baltimore, Md, USA, 1991.

[11] M. Yuan, A. De, T. Sarkar, J. Koh, and B. H. Jung, "Conditions for generation of stable and accurate hybrid TD-FD MoM solutions," IEEE Transactions on Microwave Theory and Techniques, vol. 54, no. 6, pp. 2552-2563, 2006.

[12] B. Gustavsen and A. Semlyen, "Rational approximation of frequency domain responses by vector fitting," IEEE Transactions on Power Delivery, vol. 14, no. 3, pp. 1052-1059, 1999.

[13] T. K. Sarkar and O. Pereira, "Using the matrix pencil method to estimate the parameters of a sum of complex exponentials," IEEE Antennas and Propagation Magazine, vol. 37, no. 1, pp. 4855, 1995.

[14] F. M. Tesche, "On the analysis of scattering and antenna problems using the singularity expansion technique," IEEE Transactions on Antennas and Propagation, vol. 21, no. 1, pp. 53-62, 1973.

[15] S. Makarov, Antenna and EM Modeling with MATLAB, John Wiley \& Sons, New York, NY, USA, 2002.

[16] "GEMS Software (General Electromagnetic Simulator)," http:// www.2COMU.com.

[17] C. Balanis, Antenna Theory: Analysis and Design, John Wiley \& Sons, New York, NY, USA, 1997. 

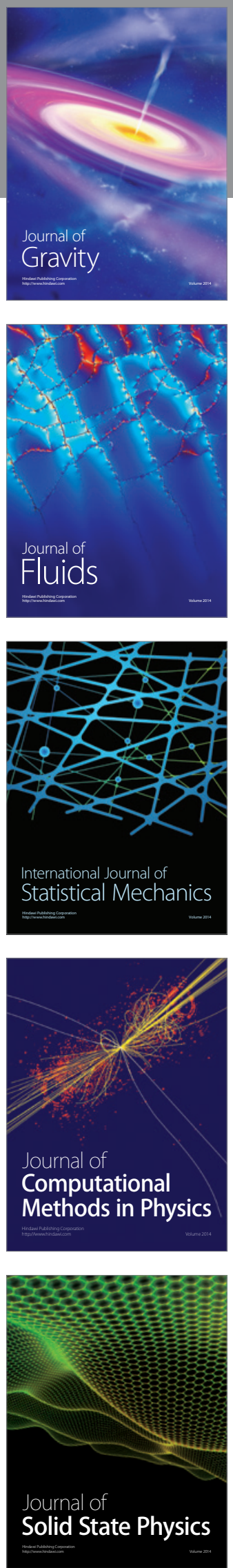

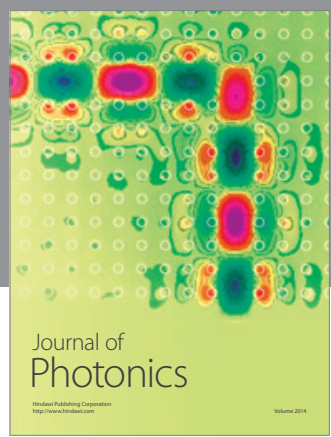

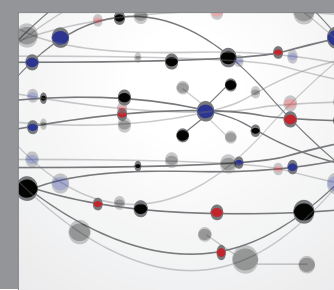

The Scientific World Journal

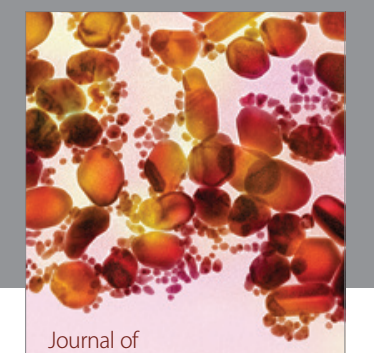

Soft Matter
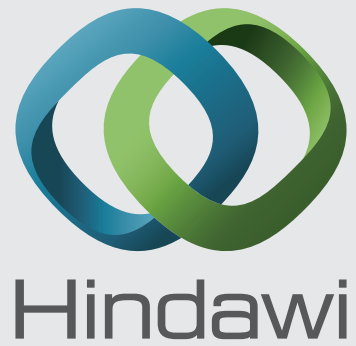

Submit your manuscripts at

http://www.hindawi.com
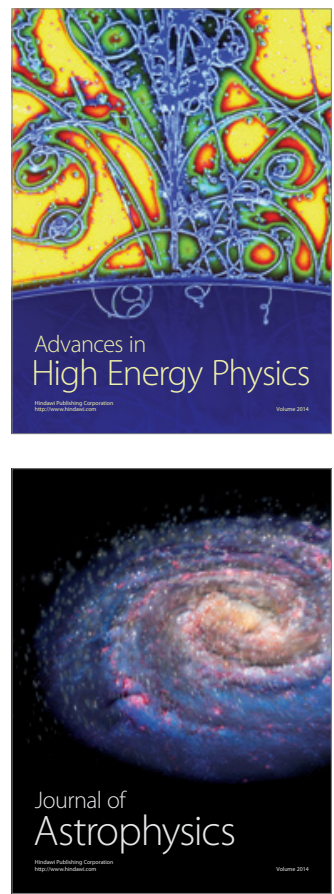
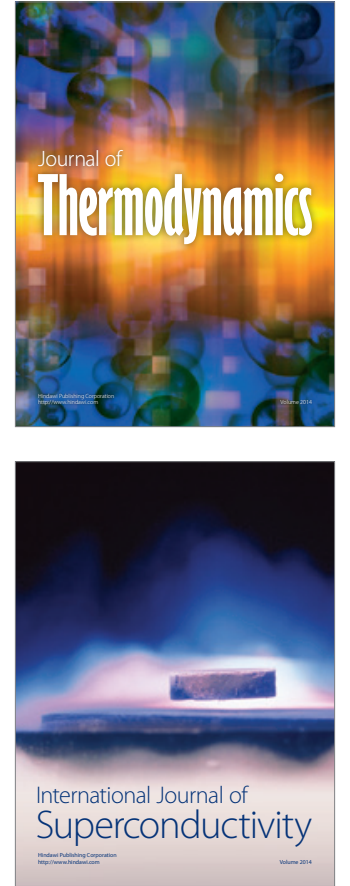
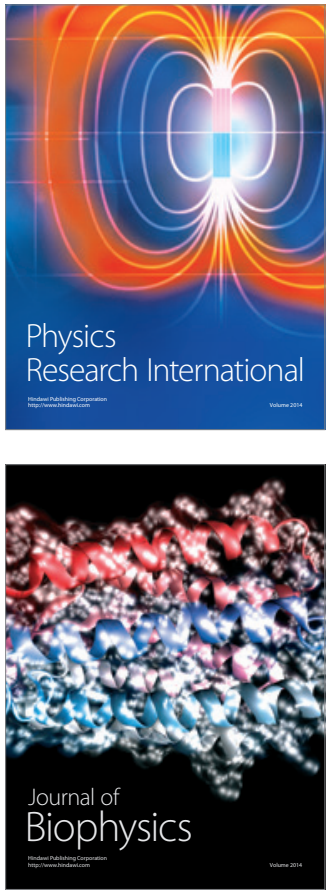
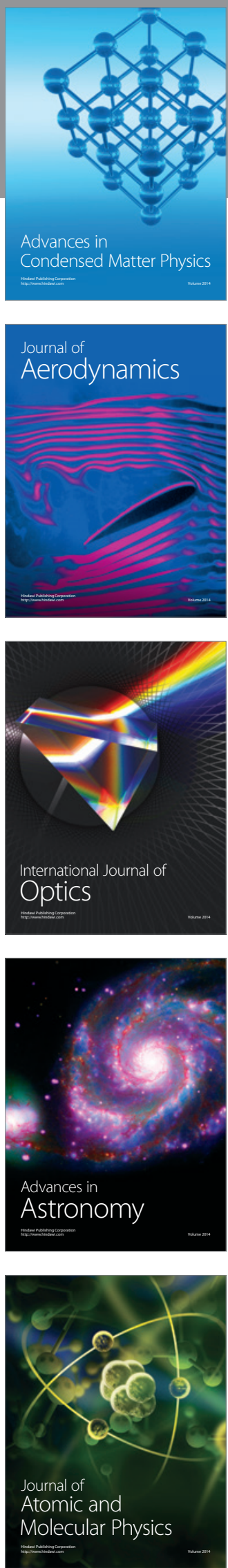